\title{
ASYMPTOTIC BEHAVIOR OF SOLUTIONS OF ORDINARY DIFFERENCE EQUATIONS
}

\author{
BY \\ CHARLES V. COFFMAN(1)
}

1. Introduction. This paper is concerned with the asymptotic behavior of solutions of ordinary difference equations with "almost constant coefficients," i.e., equations having the form

$$
y(n+1)=J y(n)+f(n, y(n)),
$$

where $y$ is a $d$-vector, $J$ is a constant $d \times d$ matrix and $f(n, y)$ is a vector-valued function which is continuous in $y$ for fixed $n$ and becomes "small" in some sense as $(n, y) \rightarrow(\infty, 0)$. Systems of this type have been studied by Perron, among others; see for example [10]. Perron has also investigated the analogous problem of the asymptotic behavior of solutions of the differential equation

$$
\frac{d y}{d t}=J y+g(t, y)
$$

where $g(t, y)$ is continuous in $(t, y)$ and becomes "small" as $(t, y) \rightarrow(\infty, 0)$, cf. [9]. These two papers of Perron serve to illustrate the parallel which exists between the two problems. It is the purpose of this paper to present analogues for difference equations of several recent developments in the theory of the asymptotic behavior of solutions of the differential equation (1.2).

The first theorem below is the analogue for difference equations of a theorem of Hartman and Wintner $[6,(*)]$ which deals with asymptotic integration of (1.2) on a logarithmic scale. The latter terminology derives from the fact that what is asserted is the following: corresponding to each eigenvalue $\lambda$ of $J$, there exist solutions $y=y(t)$ of (1.2) satisfying

$$
t^{-1} \log |y(t)|=\operatorname{Re} \lambda+o(1) \quad \text { as } t \rightarrow \infty,
$$

where $|y|$ denotes the norm of the vector $y$. The analogue of (1.3) for the difference equation (1.1) is

$$
|y(n)|^{1 / n}=|\lambda|+o(1) \quad \text { as } n \rightarrow \infty .
$$

It can be assumed that (1.1) can be written in the form

$$
y^{i}(n+1)=J_{i} y^{i}(n)+f_{i}(n, y(n)), \quad i=1,2,3,
$$

Received by the editors November 8, 1962.

(1) This research was supported by the Air Force Office of Scientific Research. 
where $y=\left(y^{1}, y^{2}, y^{3}\right)$ and the absolute values of the eigenvalues of $J_{1}, J_{2}$ and $J_{3}$ are respectively less than, equal to, and greater than $|\lambda|$. Theorem 3.1 asserts that if $y_{0}^{1}$ and $y_{0}^{2} \neq 0$ are given and if $n_{0}$ is sufficiently large, then (1.1) has a solution $y=y(n)$ such that

$$
y^{1}\left(n_{0}\right)=y_{0}^{1}, \quad y^{2}\left(n_{0}\right)=\ddot{y}_{0}^{2},
$$

(1.4) holds, and $\left|y^{i}(n)\right|=o\left(\left|y^{2}(n)\right|\right)$ as $n \rightarrow \infty$ for $i=1$,3. Results of this type, both for differential equations [9, Satz 11] and for difference equations [10, Satz 11] have been proved by Perron. For the differential equations case, Hartman and Wintner, in the theorem mentioned above, have weakened Perron's condition

(1.6) $\quad g(t, 0) \equiv 0,\left|g\left(t, y_{1}\right)-g\left(t, y_{2}\right)\right| /\left|y_{1}-y_{2}\right| \rightarrow 0$ as $\left(t, y_{1}, y_{2}\right) \rightarrow(\infty, 0,0)$ on $g(t, y)$ in (1.2) to

$$
|g(t, y)| /|y| \rightarrow 0 \text { as }(t, y) \rightarrow(\infty, 0) .
$$

With the exception that Perron's uniqueness assertion fails under this weaker hypothesis, the conclusion of their theorem contains that of Satz 11, [9] of Perron. Similarly in [10, Satz 11], Perron requires the analogue of $(1.6)$ for $f(n, y)$ in (1.1), i.e.,

$$
f(n, 0) \equiv 0,\left|f\left(n, y_{1}\right)-f\left(n, y_{2}\right)\right| /\left|y_{1}-y_{2}\right| \rightarrow 0 \text { as }\left(n, y_{1}, y_{2}\right) \rightarrow(\infty, 0,0) .
$$

He proves, then, that there exists a unique solution of (1.1) satisfying (1.5) and (1.4). In Theorem 3.1 below, with the exception of the uniqueness assertion, the conclusion of Perron's theorem is obtained under the weaker assumption

$$
|f(n, y)| /|y| \rightarrow 0 \text { as }(n, y) \rightarrow(\infty, 0) .
$$

The conclusion of Theorem 3.1 is refined in Corollary 4.1. This assertion generalizes a result of Gelfond and Kubenskaya [4] which in turn refines a classical theorem concerning the linear, scalar equation of order $d$,

$$
\left.x(n+d)+\left(a_{1}+b_{1}(n)\right) x(n+d-1)\right)+\cdots+\left(a_{d}+b_{d}(n)\right) x(n)=0 .
$$

The result of Gelfond and Kubenskaya states that if the $d$ roots $\lambda_{1}, \cdots, \lambda_{d}$ of the characteristic polynomial

$$
\lambda^{d}+a_{1} \lambda^{d-1}+\cdots+a_{d}=0
$$

of the unperturbed part of (1.10) satisfy $\left|\lambda_{1}\right|<\cdots<\left|\lambda_{d}\right|$, and if the $\left|b_{j}(n)\right|$, $j=0, \cdots, d$ have a common positive majorant $\beta(n)$ satisfying

$$
\begin{aligned}
\lim _{n \rightarrow \infty} \beta(n) & =0, \\
\beta(n+1) & \leqq \beta(n), \\
\liminf _{n \rightarrow \infty} \beta(n+1) / \beta(n) & =1,
\end{aligned}
$$


then to each $i, i=1, \cdots, d$, there corresponds a solution $x_{i}(n)$ of $(1.10)$ such that

$$
x_{i}(n+1) / x_{i}(n)=\lambda_{i}+O(\beta(n)) .
$$

In particular, Corollary 4.1 will be seen to imply that (1.14) can be weakened to

$$
\liminf _{n \rightarrow \infty} \beta(n+1) / \beta(n)>\max _{1 \leqq i<d}\left(\left|\lambda_{i}\right| /\left|\lambda_{i+1}\right|\right) .
$$

The proof given in [4] works without modification if (1.14) is replaced by (1.16)

In $\S \S 6$ and 9 below, asymptotic formulae for the solutions of (1.1) are developed. Theorem 6.1 is the analogue of $(\dagger)$ in [6], the latter is a non linear version of a theorem first proved by Dunkel [1] for the linear case of (1.2). A refinement of Dunkel's theorem for the linear case is (**) [5]; another proof for the nonlinear case has been given by Olech [8]. To my knowledge, the literature contains no theorem of this type for the difference equation (1.1) where $J$ is allowed to have characteristic numbers and elementary divisors of arbitrary multiplicities. Gelfond and Kubenskaya have, as an immediate corollary of the theorem referred to above, that when $\Sigma^{\infty} \beta(n)<\infty$, then for each $i=1, \cdots, d$, there exists a solution $x=x_{i}(n)$ of $(1.10)$ such that $x_{i}(n)=\lambda_{i}^{n}\left[1+O\left(\sum_{n+1}^{\infty} \beta(n)\right)\right]$ as $n \rightarrow \infty$. Theorem 6.1 or a theorem of Evgrabov [2], implies that if $\Sigma^{\infty}\left|b_{i}(n)\right|<\infty$, for $i=1, \cdots, d$, then for each $i,(1.10)$ has a solution satisfying $x_{i}(n)=\lambda_{l}^{n}(1+o(1))$, as $n \rightarrow \infty$. Evgrabov has proved the case of Theorem 6.1, where $f(n, y)$ is linear in $y$ for each $n, J$ is diagonal, and all of the eigenvalues of $J$ are distinct, though not necessarily of distinct absolute value. Actually the case where $J$ is diagonal but does not have distinct eigenvalues is easily reduced to the case treated by Evgrabov by a linear change of dependent variable, not affecting his condition on $f(n, y)$.

Theorems 9.1 and 9.2 also are analogues of theorems concerning differential equations (cf. (i), (ii) of [5]). In particular, the results of $\$ 9$ contain a theorem of W. Ford [3] concerning the case $d=2$ of (1.10); cf. $\$ 10$.

$\S 10$ deals with applications of the theorems of $\S \S 6$ and 9 to the scalar equation (1.10) and in particular to the second order case.

Apparently essential to the relaxation of (1.6) to (1.7) for the asymptotic ingration of (1.2) on the logarithmic scale, and to the proof of the nonlinear analogue of Dunkel's theorem is some sort of topological argument, such as, for example, the principle embodied in the theorem of Wazewski [11]. As would be expected, a topological argument also seems essential to the proofs of the analogous theorems for difference equations. Due to the discrete nature of the independent variable in this case, there appears to be no natural analogue of Ważewski's theorem for difference equations. Adequate as a substitute in the cases treated here is Lemma 3.1, whose proof is based on an argument involving the mapping degree. The lemma is, in fact, equivalent to the Brouwer fixed point theorem. 
2. Preliminary lemmas. This section contains several preliminary lemmas concerning two-dimensional systems of inequalities of the form

$$
\begin{aligned}
& u(n+1) \leqq \sigma u(n)+f(n)(u(n)+v(n)), \\
& v(n+1) \geqq \tau v(n)-f(n)(u(n)+v(n)) .
\end{aligned}
$$

It will be assumed that $f(n)$ is defined for $0,1, \cdots$, and is real and non-negative, that

and that

$$
\lim _{n \rightarrow \infty} f(n)=0
$$

$$
0 \leqq \sigma<\tau .
$$

LeMma 2.1. Let $\sigma, \tau$ be constants satisfying (2.4). Let $u(n)$ and $v(n)$ be nonnegative functions of the integral variable $n$, defined for $n_{0} \leqq n \leqq n^{0}$ and satisfying (2.1)-(2.2). Then, for any $\varepsilon>0$, there exists an $N=N(\varepsilon)$, such that if $n_{0} \geqq N$ and $u\left(n_{0}\right)<\varepsilon v\left(n_{0}\right)$, then $v(n)>0$ for $n_{0} \leqq n \leqq n^{0}$ and

$$
u(n)<\varepsilon v(n)
$$

for $n_{0} \leqq n \leqq n^{0}$.

Proof. Choose $N(\varepsilon)$ so large that both $(1+\varepsilon) f(n)<\tau$ and $(\sigma \varepsilon+f(n))(1+\varepsilon) /(\tau-f(n))(1+\varepsilon)<\varepsilon$ hold for all $n \geqq N(\varepsilon)$. When $n_{0} \geqq N(\varepsilon)$. the conclusion follows for $n_{0} \leqq n \leqq n^{0}$ by induction on $n$. If, for some $n$, $n_{0} \leqq n \leqq n^{0}, v(n)>0$, and (2.5) holds, then $v(n+1) \geqq \tau v(n)-f(n)(1+\varepsilon) v(n)>0$ and one obtains

$$
\begin{aligned}
& u(n+1) / v(n+1) \leqq[\sigma u(n)+f(n)(u(n)+v(n))] /[\tau v(n)-f(n)(u(n)+v(n))], \\
& u(n+1) / v(n+1) \leqq[\sigma \varepsilon+f(n)(1+\varepsilon)] /[\tau-f(n)(1+\varepsilon)]<\varepsilon,
\end{aligned}
$$

and so (2.5) holds for $n+1$.

It is clear from the proof just completed that when (2.3) is not assumed, the following variant of Lemma 2.1 will hold.

LEMMA 2.1'. Let $\sigma, \tau, u(n)$ and $v(n)$ be as in Lemma 2.1. Then for any $\varepsilon>0$, there exists a $\delta=\delta(\varepsilon)>0$ such that if (2.5) holds for $n=n_{0}$ and if $0 \leqq f(n) \leqq \delta(\varepsilon)$ for $n_{0} \leqq n \leqq n^{0}$, then $v(n)>0$ and $(2.5)$ holds for $n_{0} \leqq n \leqq n^{0}$.

LEMMA 2.2. Let $\sigma, \tau$ be constants satisfying (2.4). Let $u(n), v(n)$ be defined and non-negative for $n \geqq n_{0}$ and satisfy (2.1)-(2.2) and

$$
u(n)+v(n) \neq 0 \text {. }
$$

Either $(\alpha) r(n)=u(n) / v(n)$ is defined for all sufficiently large $n$ and

$$
\lim _{n \rightarrow \infty} r(n)=0
$$


or $(\beta) s(n)=v(n) / u(n)$ is defined for all sufficiently large $n$ and

$$
\lim _{n \rightarrow \infty} s(n)=0 \text {. }
$$

Proof. Assume that $(\beta)$ fails to hold. Then there exists an $\varepsilon>0$ such that (2.5) holds for arbitrarily large values of $n$. Hence, by Lemma $2.1, v(n)>0$ for all sufficiently large values of $n$ and (2.5) holds.

Let some number $\mu$ be chosen, so that $\sigma / \tau<\mu<1$. Since $(\beta)$ fails to hold, there is an $N_{0}$ so large that for all $n \geqq N_{0}, v(n)>0$, and, for some $\varepsilon>0,(2.5)$ holds, $\tau-f(n)(1+\varepsilon)>0$, and $(\sigma+f(n)) /(\tau-f(n)(1+\varepsilon))<\mu$. It follows that $r(n+1) \leqq \mu r(n)+c f(n)$ for $n \geqq N_{0}$, where $c>0$. Upon iterating this inequality one obtains

$$
r(n) \leqq \mu^{n-m} r(m)+c \sum_{l=0}^{n-m-1} \mu^{l} f(n-l-1)
$$

for $n>m \geqq N_{0}$. Let $\phi(n)$ be a positive majorant for $f(n)$, defined for $n \geqq N_{0}$, and assume that

$$
\lim _{n \rightarrow \infty} \phi(n)=0 \text { and } \phi(n+1) / \phi(n) \geqq \alpha>\sigma / \tau \text { for } n \geqq N_{0} .
$$

It may be supposed that $\mu<\alpha$. From (2.9), one has

for $n>m \geqq N_{0}$. Thus

$$
r(n) \leqq \mu^{n-m} r(m)+c \alpha^{-1} \phi(n) \sum_{l=0}^{\infty}(\mu / \alpha)^{l}
$$

$$
r(n)=O(\phi(n)) \text {. }
$$

This completes the proof of Lemma 2.2.

If $(\beta)$ holds and if $1>\mu>\sigma / \tau$, then for all sufficiently large $n$, $s(n+1) \geqq \mu^{-1} s(n)-c f(n)$, where $c>0$. Iterating this inequality yields $s(n+k) \geqq \mu^{-k}\left[s(n)-c \sum_{l=1}^{k} \mu^{l} f(n+l-1)\right]$. Since $\mu<1$, it follows from (1.8), on letting $k \rightarrow \infty$, that

$$
s(n) \leqq c \sum_{l=1}^{\infty} \mu^{l} f(n+l-1) .
$$

Thus if $\psi(n)$ is any positive majorant for $f(n)$ such that

then

$$
\lim _{n \rightarrow \infty} \psi(n)=0 \text { and } \psi(n+1) \leqq \psi(n),
$$

$$
s(n)=O(\psi(n)) .
$$

LEMMA 2.3. Let the hypothesis of Lemma 2.2 hold and assume, in addition, that for some $p, 1 \leqq p<\infty, f(n) \in l^{p}$, i.e., $\sum_{n=1}^{\infty}|f(n)|^{p}<\infty$. Then if $(\alpha)$ holds, $\sum_{=n_{0}}^{\infty}|r(n)|^{p}<\infty$ for some $n_{0}$; and if $(\beta)$ holds then $\Sigma_{n=n_{0}}^{\infty}|s(n)|^{p}<\infty$, for some $n_{0}$. 
Proof. In view of Minkowski's inequality, (2.9) and (2.12), it suffices to prove that if $f(n) \in l^{p}$, then $h(n)=\sum_{l=0}^{n-1} \mu^{l} f(n-l-1)$ and $g(n)=\sum_{l=1}^{\infty} \mu^{l} f(n+l-1)$ belong to $l^{p}$ also. Write $g(n)=\sum_{l=n}^{\infty} \mu^{l-n+1} f(l)$ and let $t=1-1 / p$. By Hölder's inequality,

Hence

$$
\begin{aligned}
|g(n)|^{p} & =\left|\sum_{l=n}^{\infty}\left(\mu^{t(l-n+1)}\right)\left(\mu^{(l-n+1) / p}\right) f(l)\right|^{p} \\
& \leqq\left(\sum_{l=n}^{\infty} \mu^{l-n+1}\right)^{t p}\left(\sum_{l=n}^{\infty} \mu^{l-n+1}|f(l)|^{p}\right) .
\end{aligned}
$$

$$
\begin{aligned}
\sum_{n=1}^{N}|g(n)|^{p} & \leqq \text { const. } \sum_{n=1}^{N} \sum_{l=n}^{\infty} \mu^{l-n+1}|f(l)|^{p} \\
& \leqq \text { const. } \sum_{l=1}^{\infty} \sum_{n=1}^{l} \mu^{l-n+1}|f(l)|^{p} \\
& \leqq \text { const. } \sum_{l=1}^{\infty}|f(l)|^{p} .
\end{aligned}
$$

If $h(n)$ is written as $h(n)=\sum_{l=0}^{n-1} \mu^{n-l-1} f(l)$ and if $t=1-1 / p$, then

$$
\begin{aligned}
|h(n)|^{p} & \leqq\left(\sum_{l=0}^{n-1} \mu^{n-l-1}\right)^{t p}\left(\sum_{l=0}^{n-l-1}|f(l)|^{p}\right) \\
& \leqq \text { const. } \sum_{l=0}^{n-1} \mu^{n-l-1}|f(l)|^{p} .
\end{aligned}
$$

The proof is completed as in the previous case.

The last two results of this section will concern the more general system

$$
\begin{aligned}
& u(n+1) \leqq \sigma(n) u(n)+f(n)(u(n)+v(n)), \\
& v(n+1) \geqq \tau(n) v(n)-f(n)(u(n)+v(n)),
\end{aligned}
$$

where $f(n)$ is non-negative and satisfies $(2.3)$ and $0 \leqq \sigma(n) \leqq \tau(n)$ for $n=0,1, \cdots$.

LEMMA 2.4. Let $u(n)$ and $v(n)$ be defined and non-negative for all $n \geqq n_{0}$, and satisfy (2.15)-(2.16), (2.6) and

$$
0<K_{0} \leqq(u(n)+v(n)) /(u(n+1)+v(n+1)) \leqq K_{1}
$$

for $n \geqq n_{0}$. Put $v(n)=\tau(n)-\sigma(n)$ and assume that $\Sigma v(n)$ is divergent. Finally let

$$
\operatorname{lub}_{0 \leqq M<\infty}\left|\sum_{k=n}^{n+M} f(k) /\left(1+\sum_{k=n}^{n+M} v(n)\right)\right| \rightarrow 0
$$


as $n \rightarrow \infty$. Set $\zeta(n)=u(n) /(u(n)+v(n))$. Then $\lim _{n \rightarrow \infty} \zeta(n)$ exists and is zero or one. Also, to any $\delta, \varepsilon, 0<\frac{1}{2} \varepsilon \leqq \delta<\varepsilon<1$, there corresponds an integer $N_{0}=N_{0}(\delta, \varepsilon)$ such that if $n_{0} \geqq N_{0}$ and $\zeta\left(n_{0}\right) \leqq \delta$, then

$$
\zeta(n)<\varepsilon \text { for all } n \geqq n_{0} .
$$

Proof. Upon multiplying (2.15) by $v(n)$ and $(2.16)$ by $-u(n)$ and adding the resulting inequalities, one obtains

$$
\begin{aligned}
(u(n+1)+v(n+1))(u(n)+v(n)) \Delta \zeta(n) \leqq & -v(n)(u(n)+v(n))^{2} \zeta(n)(1-\zeta(n)) \\
& +f(n)(u(n)+v(n))^{2}
\end{aligned}
$$

and hence

$$
\Delta \zeta(n) \leqq-K_{0} v(n) \zeta(n)(1-\zeta(n))+K_{1} f(n),
$$

where $\Delta \zeta(n)=\zeta(n+1)-\zeta(n)$. Let $c=\min \left(\frac{1}{2} K_{0} \delta(1-\varepsilon),(\varepsilon-\delta)\right)$. Choose $N_{0}=N_{0}(\delta, \varepsilon)$ so that $f(n)<\frac{1}{2} K_{1}^{-1}(\varepsilon-\delta)$ and $\sum_{k=n}^{n+M} f(k)<c K_{1}^{-1}\left(1+\sum_{k=n}^{n+M} v(k)\right)$ for any integer $M \geqq 0$ when $n \geqq N_{0}$. Let $n_{0} \geqq N_{0}, \zeta\left(n_{0}\right) \leqq \delta$, and suppose that there exists an $n_{1}>n_{0}$ such that $\zeta\left(n_{1}\right) \geqq \varepsilon$. It can be assumed that $\zeta(n)<\varepsilon$ for $n_{0} \leqq n<n_{1}$. Since $\Delta \zeta(n) \leqq K_{1} f(n)<\frac{1}{2}(\varepsilon-\delta)$ for $n \geqq N_{0}$, there exists an $m, n_{0} \leqq m<n_{1}$, such that $\zeta(n) \geqq \frac{1}{2} \delta$ for $m \leqq n \leqq n_{1}$ and $\zeta(m) \leqq \delta$. Then if $m \leqq n<n_{1}, \zeta(n)(1-\zeta(n)) \geqq \frac{1}{2} \delta(1-\varepsilon)$, so $\varepsilon-\delta \leqq \zeta\left(n_{1}\right)-\zeta(m)$ $\leqq-\frac{1}{2} K_{0} \delta(1-\varepsilon) \sum_{k=m}^{n_{1}-1} v(k)+K_{1} \sum_{k=m}^{n-1} f(k)$ but

$$
-\frac{1}{2} K_{0} \delta(1-\varepsilon) \sum_{k=m}^{n_{1}-1} v(k)+K_{1} \sum_{k=m}^{n_{1}-1} f(k)<c \leqq \varepsilon-\delta,
$$

hence a contradiction.

To complete the proof, it will be shown that if (2.19) holds for any $\varepsilon<1$, for all large $n$, then $\lim \sup \zeta(n)=0$ as $n \rightarrow \infty$. If $1>b=\lim \sup \zeta(n)>0$, then necessarily $\liminf \zeta(n)>0$, for otherwise, $\zeta(n)<b / 4$ would hold for arbitrarily large values of $n$, and hence, by the last part of the lemma $\zeta(n)<\frac{1}{2} b$ would hold for all sufficiently large $n$. It would follow then that $\zeta(n)(1-\zeta(n)) \geqq c_{0}>0$ for all sufficiently large $n$, hence $\Delta \zeta(n) \leqq-c_{0} K_{0} v(n)+K_{1} f(n)$. This yields

$$
\zeta(n+M)-\zeta(n)+c_{0} K_{0} \sum_{k=n}^{n+M} v(k) \leqq K_{1} \sum_{k=n}^{n+M} f(k)
$$

for any $M>0$ for all sufficiently large $n$. In view of the divergence of $\Sigma v(k)$, this contradicts (2.18).

If, as $n \rightarrow \infty$,

$$
v(n) \sim \text { const. } n^{-1} \text { for some positive const. }
$$

the following analogue of Lemma 2.3 holds for (2.15)-(2.16)., 
LEMMA 2.5. Let $u(n)$ and $v(n)$ be defined, non-negative, and satisfy (2.15)(2.16), (2.6) and (2.17) for $n \geqq n_{0}$. Let $\tau(n)>\sigma(n) \geqq 0$ for $n \geqq 0$, and $v(n)=\tau(n)-\sigma(n)$ satisfy (2.20). Assume that for some $p, 1 \leqq p<\infty$

$$
\sum^{\infty} n^{p-1}|f(n)|^{p}<\infty .
$$

Then the conclusion of Lemma 2.4 holds and either

$$
\sum^{\infty} n^{-1}|\zeta(n)|^{p}<\infty
$$

or

$$
\sum^{\infty} n^{-1}|1-\zeta(n)|^{p}<\infty .
$$

Proof. First it will be shown that the hypothesis of Lemma 2.4 is implied by the hypothesis of Lemma 2.5 . It is only necessary to verify that (2.20) and (2.21) imply (2.18). By Hölder's inequality,

$$
\sum_{k=n}^{n+M} f(k) \leqq\left(\sum_{k=n}^{n+M} n^{-1}\right)^{t}\left(\sum_{k=n}^{n+M} n^{p-1}|f(n)|^{p}\right)^{1 / p},
$$

where $t=1-1 / p$, but

$$
\left(\sum_{k=n}^{n+M} n^{-1}\right)^{t} \leqq 1+\sum_{k=n}^{n+M} n^{-1}
$$

Hence (2.18) follows from (2.20) and (2.21).

Suppose that $\zeta(n) \rightarrow 0$ as $n \rightarrow \infty$, then for sufficiently large $n, \zeta(n)$ satisfies

$$
\Delta \zeta(n) \leqq-K_{2} n^{-1} \zeta(n)+K_{1} f(n),
$$

where $K_{2}>0$. For some $c>0$ and for large $n$,

therefore

$$
n^{-c} \Delta n^{c}=n^{-c}\left((n+1)^{c}-n^{c}\right) \leqq K_{2} n^{-1} ;
$$

$$
\Delta\left(n^{c} \zeta(n)\right) \leqq K_{1} n^{c} f(n)
$$

for large $n$. Hence

$$
\zeta(n) \leqq\left(n_{0} / n\right)^{c} \zeta\left(n_{0}\right)+\sum_{k=n_{0}}^{n-1}(k / n)^{c} f(k)
$$

for $n_{0}$ large and $n>n_{0}$. In view of Minkowski's inequality: (2.22) follows if it is shown that

$$
\sum_{n=n_{0}+1}^{\infty} n^{-1}\left[\sum_{k=n_{0}}^{n-1}(k / n)^{c} f(k)\right]^{p}<\infty
$$


By Hölder's inequality,

Therefore

$$
\begin{aligned}
{\left[\sum_{k=n_{0}}^{n-1}(k / n)^{c} f(k)\right]^{p} } & \leqq\left[\sum_{k=n_{0}}^{n-1}(k / n)^{c}\right]^{p-1} \sum_{k=n_{0}}^{n-1}(k / n)^{c}|f(k)|^{p} \\
& \leqq \text { const. } n^{p-1} \sum_{k=n_{0}}^{N-1}(k / n)^{c}|f(k)|^{p}
\end{aligned}
$$

$$
\begin{aligned}
\sum_{n=n_{0}+1}^{N} n^{-1}\left[\sum_{k=n_{0}}^{n-1}(k / n)^{c} f(k)\right]^{p} & \leqq \text { const. } \sum_{k=n_{0}}^{N-1}|f(k)|^{p} k^{c} \sum_{n=k+1}^{N} n^{p-c-2} \\
& \leqq \text { const. } \sum_{k=n_{0}}^{N-1} k^{p-1}|f(k)|^{p}
\end{aligned}
$$

and (2.24) follows.

The proof of (2.23) when $\zeta(n) \rightarrow 1, n \rightarrow \infty$, is similar.

3. Logarithmic scale. In the system of ordinary difference equations

$$
y(n+1)=J y(n)+f(n, y(n))
$$

where $y$ and $f$ are $d$-vectors (with complex-valued components), let the $d \times d$ matrix $J$ be in a Jordan canonical form, say, with $g$ blocks. For $n=0,1,2, \cdots$, let the function $f(n, y)$ be defined and continuous in $y$ for $|y|<c$, where $c$ is some positive constant independent of $n$.

If $\lambda(1), \lambda(2), \cdots, \lambda(g)$, with $|\lambda(1)| \leqq|\lambda(2)| \leqq \cdots \leqq|\lambda(g)|$, are the eigenvalues of the blocks of $J$, let the components of the vectors $y$ and $f$ be indexed so that (3.1) can be written as a system of scalar equations of the form

(3.1 $\left.1_{j 1}\right) \quad y^{j 1}(n+1)=\lambda(j) y^{j 1}(n)+f^{j 1}(n, y(n))$,

$\left(3.1_{j k}\right) \quad y^{j k}(n+1)=\lambda(j) y^{j k}(n)+y^{j k-1}(n)+f^{j k}(n, y(n)), k=2, \cdots, h(j)$,

Let

$$
j=1, \cdots, g \text {. }
$$

$$
\rho^{1}<\rho^{2}<\cdots<\rho^{f}
$$

denote the $f$ distinct numbers among the $|\lambda(j)|$. Let some $m, 1 \leqq m \leqq f$ be chosen. An integer $j, 1 \leqq j \leqq g$, will be designated by $p=p(m), q=q(m)$, or $r=r(m)$ according as $|\lambda(j)|<\rho^{m},|\lambda(j)|=\rho^{m}$, or $|\lambda(j)|>\rho^{m}$.

In terms of the notation just introduced, define $L_{m a}=L_{m a}(y)$ for $1 \leqq m \leqq f$ and for $0<a \leqq 1$ by

$$
L_{m a}(y)=\sum_{q} \sum_{k=1}^{h(q)} a^{k}\left|y^{q k}\right| .
$$

Put $|y|=\sum_{j=1}^{f} L_{j 1}$. The norm $|f|$ of the vector $f=f(n, y)$ will be defined as 


$$
|f|=\sum_{j=1}^{q} \sum_{k=1}^{h(j)}\left|f^{j k}\right| .
$$

THEOREM 3.1. Let $f(n, y)$ satisfy

$$
|f(n, y)| /|y| \rightarrow 0 \text { as }(n, y) \rightarrow(\infty, 0) .
$$

Let

$$
\rho=\rho^{m}<1 .
$$

If $\eta$ is any number such that $0<\eta<1$ then there exist positive numbers $\delta=\delta(\eta)$ and $N=N(\eta)$ such that for any set of $\Sigma h(p)+\Sigma h(q)$ numbers $y_{0}^{p k}$ and $y_{0}^{q k}$ satisfying

$$
\begin{aligned}
\sum_{p} \sum_{k=1}^{h(p)}\left|y_{0}^{p k}\right| & \leqq \eta \sum_{q} \sum_{k=1}^{h(q)}\left|y_{0}^{q k}\right|, \\
0 & <\sum_{q} \sum_{k=1}^{h(q)}\left|y_{0}^{q k}\right|<\delta,
\end{aligned}
$$

there exists, for any $n_{0}>N$, a solution $y=y_{0}(n)$ of (3.1), defined for $n \geqq n_{0}$ and satisfying the incomplete set of initial conditions

$$
\begin{aligned}
& y^{p k}\left(n_{0}\right)=y_{0}^{p k}, \\
& y^{q k}\left(n_{0}\right)=y_{0}^{q k},
\end{aligned}
$$

and such that

$$
\lim _{n \rightarrow \infty}\left|y_{0}(n)\right|^{1 / n}=\rho .
$$

Further, if $\rho>0$, or more generally if $y_{0}(n)$ does not vanish identically for large $n$, then

$$
L_{j}\left(y_{0}(n)\right)=o\left(L_{m}\left(y_{0}(n)\right)\right) \quad \text { as } n \rightarrow \infty \text { if } j \neq m .
$$

The proof is facilitated by redefining $f(n, y)$ in the following manner:

For any $k>0$, there exists, by (3.4), a positive number $\delta_{0}=\delta_{0}(k)$, and a positive integer $N_{0}=N_{0}(k)$ such that for $n \geqq N_{0}$ and $|y| \leqq \delta_{0},|f(n, y)|<k|y|$. For some $k$, to be specified later, let $f(n, y)$ be redefined, for each $n \geqq N_{0}$, outside of $|y| \leqq \delta_{0}(k)$ so as to be continuous in $y$ and satisfy

$$
|f(n, y)|<k|y| \text { for all } y,
$$

and

$$
f(n, y) \equiv 0 \text { for }|y| \geqq \Delta,
$$

for some constant $\Delta>\delta_{0}$. Let (3.1') denote the equation (3.1) with $f$ redefined as above. Because $f$ is now defined for all $y$, for $n \geqq N_{0}$, any solution of (3.1') beginning at some $n_{0} \geqq N_{0}$ exists for all $n \geqq n_{0}$. 
When $m=f$ there is only one possible $y_{0}(n)$, since in this case (3.7) forms a complete set of initial conditions. (Unique determination of solutions of (3.1') by initial conditions can fail in the sense that two solutions with distinct initial values at $n_{0}$ can coincide for $n \geqq n_{1}>n_{0}$, however, there can obviously be only one solution of (3.1') defined for $n \geqq n_{0}$ and having a given set of initial values at $n_{0}$.) Thus, until specified otherwise, it is to be assumed that $m<f$.

From $\left(3.1_{j k}^{\prime}\right)$ it follows that if $y=y(n)$ is a solution of $\left(3.1^{\prime}\right)$ defined for $n \geqq n_{0} \geqq N_{0}$, then for any $a, 0<a \leqq 1$, and for $1 \leqq i \leqq f$, the following inequality holds

$$
\left|L_{i a}(n+1)-\rho^{i} L_{i a}(n)\right| \leqq a L_{i a}(n)+\sum_{j=q(i)} \sum_{k=1}^{h(j)}\left|f^{j k}(n, y(n))\right|,
$$

where $L_{i a}(n)=L_{i a}(y(n))$. For $1 \leqq i \leqq f$, put

$$
M_{i}(n)=M_{i a}(n)=\sum_{j=1}^{i-1} L_{j a}(n) \text { and } N_{i}(n)=N_{i a}(n)=\sum_{j=1}^{f} L_{j a}(n) \text {. }
$$

From (3.2) and (3.12) follow the inequalities

$$
\begin{gathered}
M_{i}(n+1) \leqq\left(\rho^{i-1}+a\right) M_{i}(n)+|f(n, y(n))|, \\
N_{i}(n+1) \geqq\left(\rho^{i}-a\right) N_{i}(n)-|f(n, y(n))| .
\end{gathered}
$$

Since

$$
|y(n)| \leqq a^{-d}\left(M_{i}(n)+N_{i}(n)\right),
$$

in view of (3.10), these inequalities imply

$$
\begin{aligned}
& M_{i}(n+1) \leqq\left(\rho^{i-1}+a\right) M_{i}(n)+k a^{-d}\left(M_{i}(n)+N_{i}(n)\right), \\
& N_{i}(n+1) \geqq\left(\rho^{i}-a\right) N_{i}(n)-k a^{-d}\left(M_{i}(n)+N_{i}(n)\right) .
\end{aligned}
$$

Let $a>0$ be chosen so that

$$
\rho^{i-1}+a<\rho^{i}-a
$$

for $i=m, m+1$. On applying Lemma $2.1^{\prime}$ to the system $\left(3.17_{m+1}\right)-\left(3.18_{m+1}\right)$, it follows that, given $\varepsilon>0$, if $k$ is sufficiently small and if $n_{0} \geqq N_{0}(k)$, then $N_{m+1}\left(n_{0}\right)<\varepsilon N_{m+1}\left(n_{0}\right)$ implies $N_{m+1}(n)>0$ for $n \geqq n_{0}$, and

$$
M_{m+1}(n)>\varepsilon N_{m+1}(n)
$$

for $n \geqq n_{0}$.

The following simple topological lemma will be used in proving the existence of the solution $y_{0}(n)$.

LEMMA 3.1. Let $y$ be $a d$-vector and let $A$ be a nonsingular $d \times d$ matrix. Let the d-vector valued function $g(y)$ be defined and continuous for all $y$, and satisfy

$$
g(y) \equiv 0 \text { for }|y| \geqq \gamma,
$$


for some positive constant $\gamma$. Then the mapping of the $y$-space into itself given by

$$
y_{1}=A y_{0}+g\left(y_{0}\right)
$$

is onto.

Proof. Let $F_{t}(y)=A y+t g(y), 0 \leqq t \leqq 1$. Let $y_{10}$ be arbitrary, and consider the mapping degree $a\left[y_{10}, F_{t}\right]$ of $F_{t}$ at $y_{10}$, as defined, for example, in [7]. By Theorem 5 and the remark at the end of $\S 3$ of $[7], a\left[y_{10}, F_{0}\right]= \pm 1$, hence $a\left[y_{10}, F_{1}\right]= \pm 1$ and it follows from the result just referred to that there exists a $y_{0}$ such that $y_{10}=A y_{0}+g\left(y_{0}\right)$.

Let $y_{0}^{p k}$ and $y_{0}^{q k}$ be given, satisfying (3.6. for some $\eta, 0<\eta<1$, and $\left(3.6_{2}\right)$ (with $\delta$ to be specified). Let $n_{0} \geqq N_{0}(k)$, and let $y\left(n, y_{0}^{r k}\right)$ denote the solution of $\left(3.1^{\prime}\right)$ satisfying $\left(3.7_{1}\right),\left(3.7_{2}\right)$ and

$$
y^{r k}\left(n_{0}\right)=y_{0}^{r k} \text {. }
$$

The mapping of the $\Sigma h(r)$-dimensional space of variables $y^{r k}$ into itself given by

$$
y_{1}^{r k}=y^{r k}\left(n_{0}+l, y_{0}^{r k}\right)
$$

has, for any $l>0$, the form (3.21), and because of (3.2) and (3.11), satisfies the hypothesis of Lemma 3.1. It follows that for each $l>0$, there exists a solution of $\left(3.1^{\prime}\right)$ satisfying $\left(3.7_{1}\right)$ and $\left(3.7_{2}\right)$ and with

$$
y^{r k}\left(n_{0}+l\right)=0 \text {. }
$$

For each $l>0$, let one such solution be chosen and denoted $y=y_{l}(n)$.

The auxiliary functions $M_{m+1}(n)$ and $N_{m+1}(n)$ associated with the solution $y_{l}(n)$ must satisfy

$$
M_{m+1}(n) \geqq \varepsilon N_{m+1}(n)
$$

for $n_{0} \leqq n \leqq n_{0}+l$. For if (3.23) failed to hold for some $n$ on this range, the by the choice of $k,(3.20)$ would hold at $n=n_{0}+l$, contradicting (3.22). From $\left(3.17_{m+1}\right)$ and (3.23)

$$
M_{m+1}(n+1) \leqq\left(\rho^{m}+a+k a^{-d}(1+1 / \varepsilon)\right) M_{m+1}(n),
$$

for $n_{0} \leqq n \leqq n_{0}+l$. It may be supposed that $k$ and $a$ are so small that

Then from

$$
v=\rho^{m}+a+k a^{-d}(1+1 / \varepsilon)<1 .
$$

$$
M_{m+1}(n) \leqq v^{u-n_{0}} M_{m}\left(n_{0}\right)
$$

for $n_{0} \leqq n \leqq n_{0}+l$ and (3.16), it follows that $\left|y_{l}(n)\right| \leqq v^{n-n_{0}} a^{-d}(1+1 / \varepsilon) M_{m+1}\left(n_{0}\right)$, for $n_{0} \leqq n \leqq n_{0}+l$. But $M_{m+1}\left(n_{0}\right) \leqq(1+\eta) \Sigma\left|y_{0}^{q k}\right|$, by $\left(3.6_{1}\right)$. Thus ij $\delta=\delta(\eta)$ is chosen so that $a^{-d}(1+1 / \varepsilon)(1+\eta) \delta<\delta_{0}$, then, if $y(n)=y_{l}(n)$, 


$$
|y(n)|<\delta_{0},
$$

for $n_{0} \leqq n \leqq n_{0}+l$.

From (3.25), it follows that there exists a subsequence of the $y_{l}(n)$ (which after renumeration can be supposed to be the full sequence) such that

$$
y_{0}(n)=\lim _{l \rightarrow \infty} y_{l}(n)
$$

exists for each $n \geqq n_{0}$. The auxiliary functions $M_{m+1}(n)$ and $N_{m+1}(n)$ associated with $y_{0}(n)$ satisfy (3.23) and (3.24) for all $n \geqq n_{0}$, and (3.25) holds for $y(n)=y_{0}(n)$, for all $n \geqq n_{0}$. The latter assertion implies that $y_{0}(n)$, which is certainly a solution of $\left(3.1^{\prime}\right)$, is indeed a solution of the original equation (3.1). The relations (3.16), (3.23) and (3.24), for all $n \geqq n_{0}$ imply $y_{0}(n) \rightarrow 0$ as $n \rightarrow \infty$. This in turn implies that $\left|f\left(n, y_{0}(n)\right)\right| \leqq h(n)\left(M_{m+1}(n)+N_{m+1}(n)\right)$, where $h(n) \rightarrow 0$ as $n \rightarrow \infty$. Replacing $\left|f\left(n, y_{0}(n)\right)\right|$ in $\left(3.14_{m}\right),\left(3.15_{m}\right)$ by the term on the right in the above inequality and applying Lemma 2.2 yields

$$
N_{m+1}(n)=o\left(M_{m+1}(n)\right) \text { as } n \rightarrow \infty
$$

provided $y_{0}(n) \neq 0$ for $n>n_{0}$. This completes the proof of (3.9) in case $m=1$.

Suppose $m>1$ and let $M_{m}(n), N_{m}(n)$ be the auxiliary functions associated with a solution of $\left(3.1^{\prime}\right)$ satisfying (3.7) and with the initial conditions subject to $\left(3.6_{1}\right)$. Then $M_{m}\left(n_{0}\right) \leqq \eta a^{-d} L_{m a}\left(n_{0}\right)$ and hence

$$
M_{m}(n) \leqq \eta a^{-d} N_{m}(n)
$$

for $n=n_{0} . M_{m}(n)$ and $N_{m}(n)$ satisfy $\left(3.17_{m}\right)-\left(3.18_{m}\right)$ and $a$ is such that $\left(3.19_{m}\right)$ holds. By Lemma 2.1', $k$ can be chosen so small that $n_{0} \geqq N(k)$ and (3.27) for $n=n_{0}$ imply that $N_{m}(n)$ does not vanish for $n \geqq n_{0}$ and that (3.27) holds for $n \geqq n_{0}$, since $N_{m}\left(n_{0}\right) \neq 0$ by $\left(3.6_{2}\right)$.

If $1<m<f$, let $y_{0}(n)$ be as above; if $m=f(>1)$, let $y_{0}(n)$ be the solution of $\left(3.1^{\prime}\right)$ determined by $\left(3.6_{1}\right)$. It is easy to see that in the latter case, for $n_{0}$ large and $\delta$ small, $y_{0}(n)$ satisfies (3.25), and $y_{0}(n) \rightarrow 0$ as $n \rightarrow \infty$. The solution $y_{0}(n)$ of (3.1) satisfies (3.7) so $y_{0}(n)$ does not vanish and the associated auxiliary functions $M_{m}(n)$ and $N_{m}(n)$ satisfy (3.27) for $n \geqq n_{0}$. As before, $\left|f\left(n, y_{0}(n)\right)\right|$ in $\left(3.14_{m}\right)-\left(3.15_{m}\right)$ can be replaced by $h(n)\left(M_{m}(n)+N_{m}(n)\right)$, where $h(n) \rightarrow 0$ as $n \rightarrow \infty$, and Lemma 2.1 applied to give

$$
M_{m}(n)=o\left(N_{m}(n)\right) \quad \text { as } n \rightarrow \infty .
$$

The relations (3.26) and (3.28) together if $m<f$, or (3.28) alone if $m=f(>1)$, yield (3.9). Of course (3.9) is vacuous if $f=1$.

Since $y_{0}(n) \rightarrow 0$ as $n \rightarrow \infty$, from (3.4), (3.9) and (3.12), one has, for large $n$

$$
L_{m a}(n+1) / L_{m a}(n) \leqq \rho+2 a .
$$

For some positive constant $c_{0}$, 


$$
L_{m a}(n) \leqq c_{0}(\rho+2 a)^{n} \quad \text { for } n \geqq n_{0},
$$

and this implies that

$$
\underset{n \rightarrow \infty}{\limsup }\left|L_{m a}(n)\right|^{1 / n} \leqq \rho+2 a .
$$

Since

$$
\begin{aligned}
\left|y_{0}(n)\right| & \leqq a^{-d}(1+o(1)) L_{m a}(n) \text { as } n \rightarrow \infty, \\
\limsup _{n \rightarrow \infty}\left|y_{0}(n)\right|^{1 / n} & \leqq \limsup _{n \rightarrow \infty}\left|L_{m a}(n)\right|^{1 / n},
\end{aligned}
$$

and since $a$ can be taken arbitrarily small this implies

$$
\underset{n \rightarrow \infty}{\limsup }\left|y_{0}(n)\right|^{1 / n} \leqq \rho .
$$

When $\rho>0$, the inequality

$$
L_{m a}(n+1) / L_{m a}(n) \geqq \rho-2 a
$$

leads in a similar way to

$$
\liminf _{n \rightarrow \infty}\left|y_{0}(n)\right| \geqq \rho
$$

This proves (3.8).

4. A refinement. Let $y_{0}(n)$ be a nonvanishing solution of (3.1) satisfying (3.9) (and hence (3.8)). From the proof just completed, it follows that there exists a $v, \rho^{m}<v<1$, and a constant $K$ such that $\left|y_{0}^{n}(n)\right| \leqq K v^{n}$ for $n \geqq n_{0}$. Put

$$
h(n)=\operatorname{lub}_{0<|y| \leqq K v^{n}}|f(n, y)| /|y| .
$$

It readily follows from (3.4) that $h(n) \rightarrow 0$ as $n \rightarrow \infty$. Let $\phi(n)$ and $\psi(n)$ be positive majorants for $h(n)$ satisfying respectively

$$
\lim _{n \rightarrow \infty} \phi(n)=0 \text { and } \liminf _{n \rightarrow \infty} \phi(n+1) / \phi(n)=a>\rho
$$

and

$$
\lim _{n \rightarrow \infty} \psi(n)=0 \text { and } \psi(n+1) \leqq \psi(n) \text { for all } n \geqq 0 .
$$

The assertion (3.9) of Theorem 3.1 can be refined as follows.

COROLlaRY 4.1. If $y_{0}(n)$ does not vanish for $n \geqq n_{0}$, then for $j<m$

$$
L_{j}(n) / L_{m}(n)=O(\psi(n)) \quad \text { as } n \rightarrow \infty,
$$

and for $j>m$

$$
L_{j}(n) / L_{m}(n)=O(\phi(n)) \quad \text { as } n \rightarrow \infty
$$


This result follows easily from the proof of Theorem 3.1 and the estimates (2.11) and (2.14).

5. A converse of Theorem 3.1. As a partial converse of Theorem 3.1, one has

THEOREM 5.1. Let $f(n, y)$ be as in Theorem 3.1. Let $y=y(n)(\not \equiv 0)$ for large $n$ be a solution of (3.1) defined for all sufficiently large $n$ and such that

$$
y(n) \rightarrow 0 \quad \text { as } n \rightarrow \infty,
$$

then there is an integer $m_{0}, 1 \leqq m_{0} \leqq f$, such that $\rho=\rho^{m_{0}} \leqq 1$, and (3.8) and (3.9) hold with $m=m_{0}$.

Proof. Consider the case $f>1$. Choose $a(>0)$ so small that Lemma 2.2 is applicable to $\left(3.14_{m}\right)-\left(3.15_{m}\right)$. Applying the lemma, one concludes that for each $m=1, \cdots, f$, the auxiliary functions $M_{m}(n)$ and $N_{m}(n)$ associated with $y(n)$ satisfy, as $n \rightarrow \infty$, either

$$
M_{m}(n)=o\left(N_{m}(n)\right)
$$

or

$$
N_{m}(n)=o\left(M_{m}(n)\right) \text {. }
$$

Let $m_{0}$ be either the largest integer for which $\left(5.2_{1}\right)$ holds or 1 if $\left(5.2_{1}\right)$ holds for no value of $m$. Then, the pair of relations $\left(5.2_{1}\right)$ for $m_{0}$ and $\left(5.2_{2}\right)$ for $m_{0}+1$ if $f>m_{0}>1$, the relation $\left(5.2_{2}\right)$ for $m_{0}+1$ if $m_{0}=1$, or the relation $\left(5.2_{1}\right)$ for $m_{0}$ if $m_{0}=f$, implies (3.9). As in the proof of Theorem 2.1, (3.9) implies (3.8) and (3.8), (5.1) for $m=m_{0}$ imply $\rho^{m_{0}} \leqq 1$.

The case $f=1$ is trivial.

6. Asymptotic formulae. Let some $m, 1 \leqq m \leqq f$, be chosen. Let $h_{*}$ denote the maximum multiplicity of the elementary divisors of $J$, the corresponding eigenvalues of which have absolute value $\rho^{m}$. In terms of the notations and conventions of $\$ 3$,

$$
h_{*}=\max _{q} h(q)
$$

The main result of this section is the following

THEOREM 6.1. Let $m, 1 \leqq m \leqq f$, be such that $\rho=\rho^{m}$ satisfies

$$
0<\rho<1 \text {. }
$$

Let $h_{0}$ be any number satisfying

$$
h_{*} \leqq h_{0},
$$

and let $j_{0}$ be some integer such that 


$$
0 \leqq j_{0}<h_{*} .
$$

For each $n \geqq 0$ let $f(n, y)$ be defined and continuous in $y$ for $|y| \leqq$ const. Let there exist, for each $n \geqq 0$, a scalar function $\phi(n, r)$, defined and continuous in $r$ for $|r| \leqq$ const., and having the following properties

$$
\phi(n, r) \text { is nondecreasing in } r \text { for each } n \text {, }
$$

and such that

$$
|f(n, y)| \leqq \phi(n,|y|) .
$$

For each $q$, let $l(q)$ and $k(q)$ denote the least and greatest integers, if any, satisfying

$$
1 \leqq l(q) \leqq k(q) \leqq \min \left(h(q), h_{0}-j_{0}\right), \text { and } h(q)-l(q) \leqq j_{0} .
$$

Let a set of numbers $c^{q k}$, where $l(q) \leqq k \leqq k(q)$, be given, and not all 0 . Then there exists a solution $y=y(n)$ of (3.1), defined for all large $n$, and satisfying the asymptotic formula

$$
y^{j k}(n)=o\left(n^{1-h_{0}+j_{0}} \rho^{n}\right) \quad \text { if } j \neq q,
$$

and

$$
y^{q k}(n)=\sum_{i=l(q)}^{k(q)} c^{q i} C_{n, k-i} \lambda^{n-k+i}(q)+o\left(n^{k-h_{0}+j_{0}} \rho^{n}\right),
$$

where $C_{n k}=n ! / k !(n-k) !$ is the binomial coefficient.

In particular, the hypothesis of the theorem is satisfied if (6.7) holds for some $\phi(n, r)$ having the form

$$
\phi(n, r)=\psi(n) r
$$

where

$$
\sum n^{h_{0}-1} \psi(n)<\infty .
$$

For convenience in the proof it will be assumed that for all $n \geqq 0, f(n, y)$ is defined and continuous for all $y$, and that $\phi(n, r)$ is defined, continuous, and monotone in $r$ for all $r>0$. It will be clear from the proof that this involves no loss of generality.

Let $y^{j}=\left(y^{j 1}, \cdots, y^{j h(j)}\right)$, and $f^{j}(n, y)=\left(f^{j 1}(n, y), \cdots, f^{j h(j)}(n, y)\right)$ for $j=1, \cdots, g$, (2.1) can then be written

$$
y^{j}(n+1)=J(j) y^{j}(n)+f^{j}(n, y(n)), \quad j=1, \cdots, g,
$$

where $J(j)$ is the $j$ th block of $J$ belonging to the eigenvalue $\lambda(j)$.

First the system will be transformed by a linear change of variable $y(n)=M(n) z(n)$ 
so as to diagonalize the linear part of $\left(6.12_{j}\right)$. The change of variables will be made in two steps. Let $w^{j}(n)=\left(w^{j 1}(n), \cdots, w^{j h(j)}(n)\right), j=1, \cdots, g$ be defined by

$$
\begin{aligned}
& y^{j}(n)=w^{j}(n) \quad \text { if } j \neq q, \\
& y^{q}(n)=(J(q))^{n} \lambda^{-n}(q) w^{q}(n) .
\end{aligned}
$$

Component-wise (6.13) takes the form

$$
y^{q k}(n)=\sum_{i=1}^{k} C_{n, k-i} \lambda^{-k+i}(q) w^{q i}(n) .
$$

It is immediate from $\left(6.13_{q}\right)$ that $\left(6.12_{q}\right)$ is thus transformed to

$$
w^{q}(n+1)=\lambda(q) w^{q}(n)+\lambda^{n+1}(q)(J(q))^{-n-1} f^{q}(n, y(n)) .
$$

The second step is the following, let

$$
\begin{aligned}
& w^{j k}(n)=n^{1-\beta} z^{j k}(n), \quad k=1, \cdots, h(j) \text { if } j \neq q, \\
& w^{q k}(n)=n^{k-\beta} z^{q k}(n) \quad \text { if } k<l(q) \text { or } k>k(q), \\
& w^{q k}(n)=z^{q k}(n) \quad \text { if } \quad l(q) \leqq k \leqq k(q),
\end{aligned}
$$

where $\beta=h_{0}-j_{0}$. The transformed system has the form

(6.19) $z^{q k}(n+1)=(n /(n+1))^{k-\beta} \lambda(q) z^{q k}(n)+g^{q k}(n, z(n))$, if $k<l(q)$ or $k>k(q)$,

$$
z^{q k}(n+1)=\lambda(q) z^{q k}(n)+g^{q k}(n, z(n)), \quad \text { if } \quad l(q) \leqq k \leqq k(q) .
$$

If $j \neq q, g^{j}(n, z)=(1 /(n+1))^{1-\beta_{f} j}(n, M(n) z)$. The $k$ th component of the vector $(\lambda(q))^{n+1}(J(q))^{-n-1} f^{q}(n, y)$ is $\sum_{i=1}^{k}(-1)^{k-i} C_{k-i+n n} \lambda^{k-i}(q) f^{q i}(n, y)$, so upon observing that $C_{k-i+n n}=O\left(n^{k-i}\right)$ as $n \rightarrow \infty$, it follows easily that

$$
\begin{aligned}
& \left|g^{q k}(n, z)\right| \leqq \text { const. }\left(\sum_{i=1}^{k} n^{k-i}\left|f^{q i}(n, M(n) z)\right|\right) \text { if } l(q) \leqq k \leqq k(q) \text { and } \\
& \left|g^{q k}(n, z)\right| \leqq \text { const. }\left(\sum_{i=1}^{k} n^{\beta-i}\left|f^{q i}(n, M(n) z)\right|\right) \text { if } k<l(q) \text { or } k>k(q) .
\end{aligned}
$$

Since $k(q) \leqq \beta$, one has

$$
|g(n, z)| \leqq \text { const. } n^{\beta-1}|f(n, M(n) z)| .
$$

Notice, however, that for $l(q) \leqq k \leqq k(q)$,

$$
\left|g^{q k}(n, z)\right| \leqq \text { const. } n^{k-1}|f(n, M(n) z)| .
$$

Let $s(q, k)$ be defined for $1 \leqq k \leqq h(q)$, as follows, $s(q, k)=0$ if $l(q) \leqq k \leqq k(q)$, $s(q, k)=k-\beta$ otherwise. Using (6.5), one obtains for each $q$, for $k, i=1, \cdots, h(q)$, $k-i+s(q, i) \leqq j_{0}$. Since $C_{n, k-i}=O\left(n^{k-i}\right)$, as $n \rightarrow \infty$, combining (6:16)-(6.17) 
with $\left(6.13_{q k}\right)$ yields

$$
\sum_{q} \sum_{k=1}^{h(q)}\left|y^{q k}\right| \leqq \text { const. } n^{j 0}|z| .
$$

The formulae $\left(6.13_{j}\right)$ and (6.15), together with the above imply

$$
|M(n) z| \leqq \text { const. } n^{j 0}|z| \text {. }
$$

Let $c_{0}$ and $c_{1}$ be the constants of (6.21) and (6.22), respectively. Let

$$
\psi(n, r)=c_{0} n^{\beta-1} \phi\left(n, c_{1} n^{j_{0}} r\right)
$$

by (6.21) and (6.22)

$$
|g(n, z)| \leqq \psi(n,|z|)
$$

and by $\left(6.21^{\prime}\right)$ and (6.22)

$$
\left|g^{q k}(n, z)\right| \leqq n^{k-\beta} \psi(n,|z|), \quad \text { if } l(q) \leqq k \leqq k(q) .
$$

Let two numbers $K_{0}$ and $K_{1}$ be given, with

$$
0<K_{0}<\Sigma\left|c^{q k}\right|<K_{1} \text {. }
$$

For all $n \geqq 0$, redefine $g(n, z)$ for $|z|<K_{0} \rho^{n}$ and for $|z|>K_{1} \rho^{n}$ so as to satisfy

$$
|g(n, z)| \leqq \psi\left(n, K_{1} \rho^{n}\right)
$$

and

$$
g(n, z)=0 \text { if }|y| \geqq 2 K_{1} \rho^{n} \text { or if }|y| \leqq \frac{1}{2} K_{0} \rho^{n} .
$$

Let $\left(6.18^{\prime}\right)-\left(6.19^{\prime}\right)-\left(6.20^{\prime}\right)$ denote the system (6.18)-(6.19)-(6.20) with $g(n, z)$ redefined as above. With each solution $z(n)$, of the redefined system, will be associated auxiliary functions $u(n), v(n)$ and $w(n)$ defined as follows

$$
\begin{aligned}
& u(n)=M_{m a}(n)+\sum_{q} \sum_{k>k(q)}\left|z^{q k}(n)\right|, \\
& v(n)=\sum_{q} \sum_{k=l(q)}^{k=k(q)}\left|z^{q k}(n)\right|, \\
& w(n)=N_{m+1 a}(n)+\sum_{q} \sum_{k<l(q)}\left|z^{q k}(n)\right|,
\end{aligned}
$$

where $a>0$ and $M_{m a}$ and $N_{m a}$ are defined by (3.13). One has thus $|z(n)| \leqq a^{-d}(u(n)+v(n)+w(n))$. The function $g(n, z)$, when redefined to satisfy (6.27) and (6.28), satisfies

$$
|g(n, z)| /|z| \leqq 2 K_{0}^{-1} \rho^{-n} \psi\left(n, K_{1} \rho^{n}\right) .
$$


Put

$$
\bar{\psi}(n)=2 a^{-d} K_{0}^{-1} \rho^{-n} \psi\left(n, K_{1} \rho^{n}\right) .
$$

Upon observing that $k-\beta \leqq-1$ when $k<l(q)$ and $\geqq 1$ when $k>k(q)$, it follows from $\left(6.18^{\prime}\right)-\left(6.19^{\prime}\right)-\left(6.20^{\prime}\right)$, provided $a$ is sufficiently small, that $u(n), v(n)$ and $w(n)$ satisfy

$$
\begin{aligned}
u(n+1) & \leqq(n /(n+1)) \rho u(n)+\psi(n)(u(n)+v(n)+w(n)), \\
|v(n+1)-\rho v(n)| & \leqq \psi(n)(u(n)+v(n)+w(n)), \\
w(n) & \geqq((n+1) / n) \rho w(n)-\Psi(n)(u(n)+v(n)+w(n)) .
\end{aligned}
$$

Put

$$
\begin{aligned}
& \bar{u}_{1}(n)=u(n), \quad \bar{v}_{1}(n)=v(n)+w(n), \\
& \bar{u}_{2}(n)=u(n)+v(n), \quad \bar{v}_{2}(n)=w(n), \\
& \sigma_{1}(n)=(n /(n+1)) \rho, \quad \tau_{1}(n)=\rho, \\
& \sigma_{2}(n)=\rho, \quad \tau_{2}(n)=(n+1 / n) \rho .
\end{aligned}
$$

From (6.33)-(6.34)-(6.35), one obtains

$$
\begin{aligned}
& \bar{u}_{i}(n+1) \leqq \sigma_{i}(n) \bar{u}_{i}(n)+\bar{\psi}(n)\left(\bar{u}_{i}(n)+\bar{v}_{i}(n)\right), \\
& \bar{v}_{i}(n+1) \geqq \tau_{i}(n) \bar{v}_{i}(n)-\bar{\psi}(n)\left(\bar{u}_{i}(n)+\bar{v}(n)\right),
\end{aligned}
$$

for $i=1$, 2. It follows from (6.32), (6.34), and (6.7) that $\Sigma \Psi(n)<\infty ;\left(6.37_{i}\right)$ implies that $\sigma_{i}(n)<\tau_{i}(n)$ and that $\Sigma\left[\tau_{i}(n)-\sigma_{i}(n)\right]$ is divergent, for $i=1,2$. Since $\rho>0$, the proof of Theorem 3.1 implies the existence of an integer $N_{0}$ and constants $L_{0}, L_{1}\left(0<L_{0}<L_{1}\right)$ such that if $n_{0} \geqq N_{0}$ and if $M_{m}\left(n_{0}\right)=0$, i.e., if

$$
z^{p k}\left(n_{0}\right)=0, \quad k=1, \cdots, h(p),
$$

but $z\left(n_{0}\right) \neq 0$, then $z(n)$ does not vanish for $n \geqq n_{0}$ and, for all $n \geqq n_{0}$,

$$
L_{0}<\left(M_{m}(n+1)+N_{m}(n+1)\right) /\left(M_{m}(n)+N_{m}(n)\right)<L_{1} .
$$

This implies similar inequalities for pairs, $\bar{u}_{i}(n), \bar{v}_{i}(n)$ associated with a solution $z(n)$ satisfying (6.40). It will be assumed, in fact, that $L_{0}$ and $L_{1}$ are such that if $n \geqq n_{0}$, then for such pairs

$$
L_{0}<\left(\bar{u}_{i}(n+1)+\bar{v}_{i}(n+1)\right) /\left(\bar{u}_{i}(n)+\bar{v}_{i}(n)\right)<L_{1}
$$

for $i=1,2$. Let $\varepsilon, 0<\varepsilon<1$, be given and choose $N_{1} \geqq N_{0}$, by Lemma 2.4, so that if $i=1$ or $2, n_{0} \geqq N_{1}, \bar{u}_{i}(n)$ and $\bar{v}_{i}(n)$ are defined for $n \geqq n_{0}$, and satisfy $\left(6.38_{i}\right),\left(6.39_{i}\right)$ and $(6.41)$, and if $\bar{u}_{i}\left(n_{0}\right)<\frac{1}{2} \varepsilon_{i}\left(\bar{u}_{i}\left(n_{0}\right)+\bar{v}_{i}\left(n_{0}\right)\right)$, then 


$$
\bar{u}_{i}(n)<\varepsilon_{i}\left(\bar{u}_{i}(n)+\bar{v}_{i}(n)\right)
$$

for $n \geqq n_{0}$, where $\varepsilon_{1}=\frac{1}{2} \varepsilon_{2}=\frac{1}{2} \varepsilon$.

Let $n_{0} \geqq N_{1}$ be fixed. By Lemma 2.1 there exists, for each $v \geqq n_{0}$, a solution $z_{v}(n)$ satisfying $(6.40)$

$$
\begin{aligned}
z^{q k}\left(n_{0}\right) & =0, \quad k>k(q), \\
z^{q k}(v) & =c^{q k} \lambda^{v}(q), \quad l(q) \leqq k \leqq k(q)
\end{aligned}
$$

and

$$
z^{j k}(v)=0 \text { if } j=r \text {, or if } k<l(q) .
$$

The auxiliary functions $\bar{u}_{i}(n), \bar{v}_{i}(n), i=1,2$, satisfy (6.41) for $n \geqq n_{0}$, hence, since $\bar{u}_{1}\left(n_{0}\right)=0,\left(6.42_{1}\right)$ holds, for $n \geqq n_{0}$. On the other hand, $\bar{v}_{2}(v)=0$, so it must be the case that

$$
\bar{u}_{2}(n) \geqq \varepsilon\left(\bar{u}_{2}(n)+\bar{v}_{2}(n)\right),
$$

for $n_{0} \leqq n<v$. The relations (6.42) and (6.46) for $n_{0} \leqq n<v$, imply, if $\varepsilon$ is sufficiently small,

$$
u(n) \leqq \frac{1}{2} \delta v(n) \text { and } w(n) \leqq \frac{1}{2} \delta v(n)
$$

for $n_{0} \leqq n \leqq v$, where $\delta=\delta(\varepsilon)>0$. The inequalities (6.47), combined with (6.34), yield,

$$
|v(n+1)-\rho v(n)| \leqq \Psi(n)(1+\delta) v(n),
$$

for $n_{0} \leqq n \leqq v$. If $n$ is on the latter range, (6.48), (6.44) and (6.25) imply

$$
K_{0} L^{-1}<\rho^{-n} v(n)<K_{1} L
$$

where $L=\exp$ (const. $\sum_{n=n_{0}}^{\infty} \Psi(n)$ ), the const. (>0) depending only on $\rho, \delta$, and a bound for $\psi(n)$. The relations (6.47) and (6.49) imply in particular that $z_{v}\left(n_{0}\right)$ is bounded independently of $v$. From (6.20), (6.25) and (6.49) one has, if $l(q) \leqq k \leqq k(q)$,

$$
\left|z_{v}^{q k}(n+1)-\lambda(q) z_{v}^{q k}\right| \leqq n^{k-\beta} \bar{\psi}(n)(1+\delta) K_{1} L \rho^{n},
$$

for $n_{0} \leqq n \leqq v$. It follows that

$$
\begin{aligned}
\left|\lambda^{n-v}(q) z_{v}^{q k}(v)-z_{v}^{q k}(n)\right| & \leqq \sum_{i=1}^{v-1} \rho^{-i+n-1}\left|z_{v}^{q k}(i+1)-\lambda(q) z_{v}^{q k}(i)\right| \\
& \leqq \rho^{n-1} K_{1} L(1+\delta) \sum_{i=n}^{\infty} i^{k-\beta} \Psi(i) .
\end{aligned}
$$


Because of (6.44) this yields

$$
\left|z_{v}^{q k}(n)-c^{q k} \lambda^{n}(q)\right| \leqq \rho^{n-1} K_{1} L(1+\delta) \sum_{i=n}^{\infty} i^{k-\beta} \psi(i),
$$

if $n_{0} \leqq n \leqq v$, for $l(q) \leqq k \leqq k(q)$.

Continuous dependence on initial conditions for solutions of $\left(6.18^{\prime}\right)-\left(6.19^{\prime}\right)$ $-\left(6.20^{\prime}\right)$ and the boundedness of $z_{v}\left(n_{0}\right)$ for $v \geqq n_{0}$ implies the existence of an increasing sequence of integers $v(k)$ such that

$$
z(n)=\lim _{k \rightarrow \infty} z_{v(k)}(n)
$$

exists for each $n \geqq n_{0}$. The function $z(n)$ defined by (6.51) is a solution of $\left(6.18^{\prime}\right)-\left(6.19^{\prime}\right)-\left(6.20^{\prime}\right)$ and the associated auxiliary functions $u(n), v(n)$ and $w(n)$ are the limits of the respective auxiliary functions associated with the $z_{v(k)}(n)$. It follows that the auxiliary functions for $z(n)$ satisfy (6.47) for all $n \geqq n_{0}$. In view of the definitions $\left(6.36_{1}\right),\left(6.36_{2}\right)$, it follows, on applying Lemma 2.4 to the systems $\left(6.38_{i}\right)-\left(6.39_{i}\right), i=1,2$, that

$$
u(n)=o(v(n)), \quad w(n)=o(v(n)), \quad \text { as } n \rightarrow \infty .
$$

Finally (6.50), for each $v \geqq n_{0}$, implies that $z(n)$ satisfies

$$
z^{q k}(n)=c^{q k}(\lambda(q))^{n}+o\left(n^{k-\beta} \rho^{n}\right), \quad \text { as } n \rightarrow \infty,
$$

for $l(q) \leqq k \leqq k(q)$. The latter relation, together with (6.52) and (6.26) imply that for large $n, K_{0}<\rho^{-n}|z(n)|<K_{1}$, so that $z(n)$ is, for large $n$, a solution of the original system (6.18)-(6.19)-(6.20).

It readily follows from $(6.52),(6.53)$ and the definition of $M(n)$, that the solution $y(n)=M(n) z(n)$ of (6.12) satisfies (6.9).

7. The linear case. The condition on $f(n, y)$ in Theorem 6.1 is essentially invariant under a change of dependent variable of the form $y(n)=A y(n)$, where $A$ is a nonsingular constant matrix. Hence, to check the condition it is not necessary to have $J$ in a Jordan normal form. If however $J$ is in such a form, and in particular when $f(n, y)$ is linear in $y$, i.e., in the notation of $\S 3$,

$$
f^{j k}(n, y)=\sum_{\mu=1}^{g} \sum_{\nu=1}^{h(\alpha)} \gamma_{j k \mu \nu}(n) y^{\mu \nu}
$$

then the condition on $f(n, y)$ can be relaxed somewhat. Suppose that for some pair $(q k)=\left(q_{0} k_{0}\right)$, one has

$$
c^{q_{0} k_{0}}=1, \quad c^{q k}=0 \quad \text { if } \quad(q k) \neq\left(q_{0} k_{0}\right) .
$$

Take $j_{0}=h\left(q_{0}\right)-k_{0}$, so that $\beta=h_{0}-h\left(q_{0}\right)+k_{0}$. Let $l\left(q_{0}\right)=k\left(q_{0}\right)=k_{0}$. If $\beta$ is not an integer, let $k(q)$ and $l(q)$ remain undefined for $q \neq q_{0}$, if $\beta$ is an integer, 
and $h(q) \geqq \beta$, let $l(q)=k(q)=\beta$. For those $q$ for which $h(q)<\beta, l(q)$ and $k(q)$ will be undefined in any case. Make the change of variable $y(n)=M(n) z(n)$, where $M(n)$ is as in $\S 6$, with $\beta, l(q)$ and $k(q)$ as above. The function $g(n, z)$ in the resulting system (6.18)-(6.19)-(6.20) will have the form

$$
g^{j k}(n, z)=\sum_{\mu=1}^{g} \sum_{v=1}^{h(\mu)} \tilde{g}_{j h \mu v}(n) z^{\mu \nu}
$$

The proof given in $\S 6$ will work if

$$
\Sigma\left|\tilde{g}_{j k \mu v}(n)\right|<\infty, \quad \text { for each }(j k \mu v),
$$

and

$$
\sum_{n}^{h_{0}-h\left(q_{0}\right)}\left|\tilde{g}_{q_{0} k_{0} \mu \nu}\right|<\infty, \quad \text { for each }(\mu \nu)
$$

This will be the case if

$$
\sum\left|\gamma_{j k \mu \nu}(n)\right| n^{\varepsilon(\alpha \beta)-\varepsilon(j k)+\varepsilon(\alpha)\left(h_{0}-k(q)\right)}<\infty
$$

holds for each $(j k \mu v)$, where $\varepsilon(j k)$ is $k$ or 1 according as $j=q$ or $j \neq q$, and $\varepsilon(j)$ is 1 or 0 according as $j=q_{0}$ or $j \neq q_{0}$. Thus when (7.1) and (7.6) hold, (3.1) has a solution $y=y(n)$ satisfying (6.9), where $c_{q k}$ are as in (7.2).

For the $d$ th order scalar difference equation

$$
\Delta^{d} x(n)+a_{1}(n) \Delta^{d-1} x(n)+\cdots+a_{d}(n) x(n)=0,
$$

where $\Delta^{k} x(n)=\sum_{i=0}^{k}(-1)^{k-i} C_{k i} x(n+i)$, the above remark has the following implication. If

$$
\sum n^{j-1}\left|a_{j}(n)\right|<\infty
$$

for $j=1, \cdots, d$, then for each $k_{0}=0,1, \cdots, d-1(7.7)$ has a solution $x=x(n)$ satisfying

$$
\Delta^{k} x(n)=C_{n k_{0}-k}+o\left(n^{k_{0}-k}\right) \quad \text { for } 0 \leqq k \leqq k_{0},
$$

and

$$
\Delta^{k} x(n)=o\left(n^{k_{0}-k}\right) \quad \text { for } k_{0}<k \leqq d-1 .
$$

The equation (7.7) is equivalent to the case of (1.10), where the roots of the characteristic equation satisfy $\lambda_{1}=\lambda_{2}=\cdots=\lambda_{d}=1$. Any case of (1.10) for which the characteristic equation has a single nonzero root of multiplicity $d$ may be reduced to this case by a change of variable. If (7.7) is written in the form (1.10), then for the conclusion above to remain valid it is in general necessary to require

for $j=1, \cdots, d$.

$$
\sum n^{d-1}\left|b_{j}(n)\right|<\infty,
$$


8. A converse of Theorem 6.1. Theorem 6.1 admits the following partial converse:

THEOREM 8.1. Let $\rho=\rho^{m}$ satisfy (6.2). Let $h_{*}$ be defined by (6.1) and let $h_{0}$ satisfy (6.3). For each $n \geqq 0$, let $f(n, y)$ be defined and continuous in $y$, for $|y| \leqq$ const. $(\neq 0)$. Let there exist a function $\phi(n, r)$ defined for all $n \geqq 0$ and for small $r \geqq 0$, continuous in $r$ for each $n$, and satisfying (6.7). Let $\phi(n, r)$ have the form (6.10), where (6.11) holds, or let $\phi(n, r)$ satisfy, in addition to (6.5),

$$
\phi(n, r) \text { is nondecreasing in } n \text { for each fixed } r \text {, }
$$

and in place of (6.6), for some $\delta, 0<\delta<\rho$

$$
\Sigma(\rho-\delta)^{-n^{h_{0}-1}} \phi\left(n, \text { const. }(\rho-\delta)^{n}\right)<\infty, \quad \text { for every const. }>0 .
$$

Let $y=y(n)$ be a solution of (3.1) satisfying (3.8) and (3.9). Then for some $j_{0}, 0 \leqq j_{0}<h_{*}$, and some constants $c^{4 k}$ not all $0, y$ satisfies the asymptotic formula $\left(6.9_{1}\right)-\left(6.9_{2}\right)$.

An argument similar to one used in the proof below shows that (6.5), (8.1) and (8.2) imply (6.6).

Proof. The first step of the proof consists of showing that (3.8) and (3.9) imply

$$
\sum n^{h_{0}-1}|f(n, y(n))| /|y(n)|<\infty .
$$

If (6.7), (6.10) and (6.11) hold, and if $y(n)$ is any solution of (3.1) defined for all large $n$ and such that $y(n) \neq 0$ for large $n$, then (8.3) is immediate. It will be shown that (8.3) is valid if $y(n)$ satisfies (3.8) and (3.9) when (6.7), (6.5), (8.1) and (8.2) hold.

Let $\delta>0$ be such that (8.2) holds and let $\alpha(n)$ be the largest integer not exceeding $[\log (\rho-\delta)]^{-1} \log L_{m a}(n)$. It follows that

$$
L_{m a}(n) \leqq(\rho-\delta)^{\alpha(n)}
$$

and

$$
\left(L_{m a}(n)\right)^{-1} \leqq(\rho-\delta)^{-(\alpha(n)+1)} .
$$

From (3.8) and (3.9) one has that for large $n$,

$$
\alpha(n) \leqq n .
$$

By (3.3) and (3.9) there exists a positive constant $K$ such that

$$
L_{m a}(n) \leqq|y(n)| \leqq K L_{m a}(n),
$$

so by (6.5), (8.1), (8.4), (8.5) and (8.6), 


$$
|f(n, y(n))| /|y(n)| \leqq(\rho-\delta)^{-(\alpha(n)+1)} \phi\left(\alpha(n), K(\rho-\delta)^{\alpha(n)}\right) .
$$

It follows from (3.8) and (8.7) that $\alpha(n) \rightarrow \infty$ as $n \rightarrow \infty$, hence by (8.2), the term on the right of (8.8) tends to zero as $n \rightarrow \infty$. In view of (3.12) and (8.7), this implies that there exists a $c>0$ such that for all sufficiently large $n, \Delta \log L_{m a}(n)<-c$. This in turn implies that there exists a positive integer $\Re$ such that the integral valued function $\alpha(n)$ takes any given value at most $\boldsymbol{\Omega}$ times, and that $\alpha(n) \sim$ const. $n$ as $n \rightarrow \infty$. These facts and (8.2) imply the convergence of

$$
\Sigma(\rho-\delta)^{-\alpha(n)} n^{h_{0}-1} \phi\left(\alpha(n), K(\rho-\delta)^{\alpha(n)}\right),
$$

which together with (8.8) implies (8.3).

Let $f(n, y)=\left(f^{1}(n, y), \cdots, f^{d}(n, y)\right)$, and let $y=\left(y^{1}, \cdots, y^{d}\right)$. For large $n$, define a matrix $\Gamma(n)$ as follows

$$
\gamma_{j k}(n)=f^{j}(n, y(n)) \bar{y}^{k}(n) /|y(n)|^{2},
$$

where the bar denotes complex conjugation. By (8.3), $\Gamma(n)$ satisfies

$$
\sum n^{h_{0}-1}|\Gamma(n)|<\infty,
$$

so Theorem 6.1 is applicable to the linear system

$$
y(n+1)=(J+\Gamma(n)) y(n) .
$$

The function $y(n)$, being a solution of (3.1), satisfies (8.11). The result follows by applying Theorem 6.1 to (8.11) and using the superposition principle.

9. Variants for the linear case of (3.1). When $f(n, y)$ has the form (7.1), one can obtain results concerning the asymptotic behavior of solutions of (3.1) under conditions different from those in Theorem 3.1. Theorems 9.1 and 9.2 below are, respectively, the analogues of (i) and (ii) of [5].

THEOREM 9.1. In (3.1) let $f(n, y)$ be linear

$$
f^{i}(n, y)=\sum_{j=1}^{d} \gamma_{i j}(n) y^{j}, \quad i=1, \cdots, d,
$$

where $y=\left(y^{1}, \cdots, y^{d}\right)$ and $f=\left(f^{1}, \cdots, f^{d}\right)$. Let $\lambda(1), \cdots, \lambda(d)$ be the $d$ eigenvalues of $J$, and let there exist an $m, 1 \leqq m \leqq d$, with the property that $\lambda(m) \neq 0$, and $i \neq m$ implies $|\lambda(i)| \neq|\lambda(m)|$. Finally, for some $\sigma$ on the range $1 \leqq \sigma \leqq 2$, let

$$
\stackrel{\infty}{\Sigma}^{\infty}\left|\gamma_{i j}(n)\right|^{\sigma}<\infty
$$

for each $i, j=1, \cdots, d$. Then (3.1) possesses a solution $y(n)=\left(y^{1}(n), \cdots, y^{d}(n)\right)$, defined for all large $n$, such that as $n \rightarrow \infty$ 


$$
y^{m}(n) \sim \prod^{n-1}\left(\lambda(m)+\gamma_{m m}(n)\right)
$$

and

$$
\left|y^{i}(n)\right|=o\left(\left|y^{m}(n)\right|\right) \quad \text { if } i \neq m .
$$

Proof. The existence of $y(n)(\not \equiv 0)$ satisfying (9.4) follows from Theorem 3.1. Lemma 2.3 and the proof of Theorem 3.1 imply in fact that $\left|y^{i}(n)\right| /\left|y^{m}(n)\right| \in l^{\sigma}$ for $i \neq m$. The latter relation and $1 \leqq \sigma \leqq 2,1 / \sigma+1 / \sigma^{\prime}=1$ give

$$
\left|y^{i}(n)\right| /\left|y^{m}(n)\right| \in l^{\sigma^{\prime}} \text { for } i \neq m \text {. }
$$

One can write

$$
y^{m}(n+1) / y^{m}(n)=\left(\lambda(m)+\gamma_{m m}(n)\right)(1+\phi(n))
$$

where

$$
\phi(n)=\left(\sum_{i \neq m} \gamma_{m i}(n) y^{i}(n) / y^{m}(n)\right) /\left(\lambda(m)+\gamma_{m m}(n)\right)
$$

By Hölder's inequality $\sum_{i \neq m} \gamma_{m i}(n) y^{i}(n) / y^{m}(n) \in l^{1}$, and hence so is $\phi(n)$. Thus the infinite product $\prod(1+\phi(n))$ is convergent and (9.3) follows from (9.6).

THEOREM 9.2. Let $h_{*}$ be defined by (6.1); $q_{0}$ a particular value of $q$, where $\rho=|\lambda(q)|>0 ; k_{0}$ an integer satisfying $1 \leqq k_{0} \leqq h\left(q_{0}\right)$. Let $h_{0}$ be a number satisfying (6.3) and let

$$
\beta-k \neq 0 \text { if } 1 \leqq k \leqq h(q) \text { and } q \neq q_{0} \text {, }
$$

where $\beta=h_{0}-h_{0}\left(q_{0}\right)+k_{0}$. Let $f(n, y)$ satisfy $(7.1)$, and for some $\sigma, 1 \leqq \sigma \leqq 2$, let

$$
\stackrel{\infty}{\Sigma}^{\infty}\left|\gamma_{j k \mu \nu}(n)\right|^{\sigma} n^{\sigma\left\{\varepsilon(\mu v)-\varepsilon(j k)+\varepsilon(\alpha)\left(h_{0}-h\left(q_{0}\right)\right)\right\}+\sigma-1}<\infty,
$$

for each $(j k \mu v)$, where $\varepsilon(j k), \varepsilon(j)$ are as defined in \$7. Then (3.1), (7.1) has a solution which satisfies, as $n \rightarrow \infty$

(9.9 $) y^{q k}(n)=O\left(n^{k-\beta} \rho^{n}|\Lambda(n)|\right)$ if $q \neq q_{0}$ or if $k<k_{0}$,

$\left(9.9_{2}\right) y^{q k}(n)=C_{n, k-k_{0}}(\lambda(q))^{n-k+k_{0}} \Lambda(n)+o\left(n^{k-\beta} \rho^{n}|\Lambda(n)|\right)$ if $q=q_{0}$ and $k \geqq k_{0}$,

and

$$
y^{j k}(n)=o\left(n^{1-\beta} \rho^{n}|\Lambda(n)|\right) \quad \text { if } j \neq q,
$$

where

$$
\begin{gathered}
\Lambda(n)=\prod_{m=n_{0}}^{n-1}\left(1+\sum_{i=1}^{k_{0}} \sum_{v=k_{0}}^{h\left(q_{0}\right)}(-1)^{k_{0}-i} C_{k_{0}-i+m} C_{m, v-k_{0}}\right. \\
\left.\cdot \lambda\left(q_{0}\right)^{2 k_{0}-v-i-1} g_{q_{0} i q_{0} v}(m)\right) .
\end{gathered}
$$


Proof. Make the change of variables of $\$ 7$. One obtains thus a system of the form (6.18)-(6.19)-(6.20), where $g(n, z)$ satisfies (7.3). From (9.8) it follows that for each $(j k \mu v)$

$$
\sum^{\infty} n^{\sigma-1}\left|g_{j k \mu v}\right|^{\sigma}<\infty
$$

and that for each $(\mu v)$

$$
\sum^{\infty} n^{\sigma\left(h_{0}-h\left(q_{0}\right)\right)+\sigma-1}\left|g_{q_{0} k_{0} \mu}\right|^{\sigma}<\infty .
$$

The proof of Theorem 6.1, with Lemma 2.4 replaced by Lemma 2.5, implies that the system $(6.18)-(6.19)-(6.20)$ has a solution $z(n)$ such that $z^{q_{0} k_{0}}(n) \neq 0$ for large $n$,

$$
\left|z^{j k}(n)\right|=o\left|z^{q_{0} k_{0}}(n)\right| \text { as } n \rightarrow \infty,
$$

and

$$
\sum^{\infty} n^{-1}\left|z^{j k}(n) / z^{q_{0} k_{0}}(n)\right|<\infty .
$$

(Because of $(9.7), v(n)$, as defined by (6.30) satisfies $v(n)=\left|z^{q_{0} k_{0}}(n)\right|$, see the definition of $l(q), k(q)$ in $\S 7$.) The case $q_{0} k_{0}$ of $(6.20)$ can be written

$$
z^{q_{0} k_{0}}(n+1)=\left(\lambda(q)+\tilde{g}_{q_{0} k_{0} q_{0} k_{0}}(n)+\phi(n)\right) z^{q_{0} k_{0}}(n),
$$

where by (9.12), (9.14) and Hölder's inequality

$$
\sum^{\infty} n^{h_{0}-h\left(q_{0}\right)}|\phi(n)|<\infty .
$$

An argument like that used in the proof of Theorem 9.1 gives

$$
z^{q_{0} k_{0}}(n) \sim\left(\lambda\left(q_{0}\right)\right)^{n} \prod^{n-1}\left(1+\left(\lambda\left(q_{0}\right)\right)^{-1} \tilde{g}_{q_{0} k_{0} q_{0} k_{0}}(m)\right)
$$

in view of (9.16), this can be sharpened to

$$
z^{q_{0} k_{0}}(n)=\left(\lambda\left(q_{0}\right)\right)^{n} \prod^{n-1}\left(1+\left(\lambda\left(q_{0}\right)\right)^{-1} \tilde{g}_{q_{0} k_{0} q_{0} k_{0}}(m)\right)\left(1+o\left(n^{h\left(q_{0}\right)-h_{0}}\right)\right) .
$$

Since

$$
\tilde{g}_{q_{0} k_{0} q_{0} k_{0}}(n)=\sum_{i=1}^{k_{0}} \sum_{v=k_{0}}^{h\left(q_{0}\right)}(-1)^{k-i} C_{k_{0}-i+n, n} C_{n, v-k_{0}}(\lambda(q))^{2 k_{0}-v-i} g_{q i \mu v}(n),
$$

the formulae (9.9) follow from (9.13), (9.17) and the definition of the change of variable $y(n)=M(n) z(n)$ of $\S 7$.

10. Applications to the scalar difference equation. The following theorem of Evgrabov [2], is an immediate corollary of Theorem 6.1. 
THEOREM 10.1. Let the characteristic equation (1.11) of the difference equation (1.10) have d distinct, nonzero roots $\lambda_{1}, \cdots, \lambda_{d}$. For $j=1, \cdots, d$ let $\Sigma^{\infty}\left|b_{j}(n)\right|$ $<\infty$. Then for each $j=1, \cdots, d,(1.10)$ has a solution $x_{j}=x_{j}(n)$ satisfying $x_{j}(n)=\lambda_{j}^{n}(1+o(1))$ as $n \rightarrow \infty$.

The remainder of this section will be concerned with the second order difference equation

$$
x(n+2)+a_{1}(n) x(n+1)+a_{2}(n) x(n)=0 .
$$

Rather than assuming that (10.1) is of the "almost constant coefficient" type it will be assumed that for some $n_{0}$,

$$
a_{1}(n) \neq 0 \quad \text { for } n \geqq n_{0}
$$

and that,

$$
\lim _{n \rightarrow \infty} 4 a_{2}(n) / a_{1}(n) a_{1}(n-1)=p
$$

exists. For $n>n_{0}$, let $\alpha(n)$ be defined by

$$
4 a_{2}(n) / a_{1}(n) a_{1}(n-1)=p+\alpha(n) .
$$

THEOREM 10.2. Let (10.2) hold for some $n_{0}$ and let there exist a constant $p$ such that (10.3), (10.4) and $\Sigma|\alpha(n)|<\infty$ hold. Assume that $p$ is neither 0 nor 1 and put $v=(1-p)^{1 / 2}$. Then $(10.1)$ has solutions $x_{ \pm}(n)$ satisfying respectively, as $n \rightarrow \infty$,

$$
x_{ \pm}(n) \sim\left(-\frac{1}{2}\right)^{n}\left(\prod^{n-1} a_{1}(k)\right)(1 \pm v)^{n}
$$

The case $p=1$ generalizes the almost constant coefficient case of (10.1), where the characteristic equation has a nonzero double root. For this case one has

THEOREM 10.3. Let (10.2) and (10.3) hold. Assume that $p=1$ and that $\alpha(n)$ defined by (10.4) satisfies $\Sigma n|\alpha(n)|<\infty$. Then (10.1) has solutions $x_{1}(n)$ and $x_{2}(n)$ satisfying respectively, as $n \rightarrow \infty$

$$
x_{1}(n) \sim\left(-\frac{1}{2}\right)^{n} \prod^{n-1} a_{1}(k) \text { and } x_{2}(n) \sim\left(-\frac{1}{2}\right)^{n} n \prod^{n-1} a_{1}(k) .
$$

Proof of Theorems 10.2 and 10.3 Put

$$
x(n)=\left(-\frac{1}{2}\right)^{n}\left(\prod_{k=u_{0}}^{n-1} a_{1}(k)\right) u(n) \quad \text { for } n>n_{0} .
$$

Then (10.1) is transformed to

$$
u(n+2)-2 u(n+1)+(p+\alpha(n)) u(n)=0 .
$$


The characteristic equation of (10.6), when $p \neq 1$ has the roots $(1 \pm v)$ and Theorem 10.2 follows from Theorem 10.1 and (10.5). When $p=1,(10.6)$ may be written $\Delta^{2} u(n)+\alpha(n) u(n)=0$, i.e., the case $d=2$ of (7.7) and Theorem 10.3 follows from (10.5) and the remarks in $\$ 7$. When (10.2) holds and $p=0$, then (10.5) and Theorem 6.1 applied to (10.6), imply that (10.1) has a solution $x_{1}(n)$ which satisfies as $n \rightarrow \infty$

$$
x_{1}(n) \sim(-1)^{n} \prod^{n-k} a_{1}(k)
$$

By Theorem $3.1(10.2)$ also has a solution $x_{2}=x_{2}(n)$ such that as $n \rightarrow \infty$

$$
x_{2}(n)=o\left(a^{n}\left|x_{1}(n)\right|\right)
$$

for any $a, 0<a<1$, Theorem 6.1 yields no additional information concerning this solution.

For the "almost constant coefficient" case of (10.1), (10.2) and $\Sigma\left|b_{i}(n)\right|<\infty$ for $i=1,2$, (or $\Sigma^{\infty} n\left|b_{i}(n)\right|<\infty$ for $i=1,2$ ), where $b_{i}(n)=a_{i}(n)-\lim _{n \rightarrow \infty} a_{i}(n)$, imply $\Sigma|\alpha(n)|<\infty$, (or $\left.\Sigma^{\infty} n|\alpha(n)|<\infty\right)$. Thus with the possible exception of the case where $\lim _{n \rightarrow \infty} a_{1}(n)=0$, the applications of Theorem 6.1 to the almost constant coefficient case of (10.1) are contained in Theorems 10.2 and 10.3 and the remark above concerning the case $p=0$. If $\lim _{n \rightarrow \infty} a_{1}(n)=0$, then the characteristic equation has two nonzero roots $\lambda$, and $-\lambda$, or a double root 0 . The former case is contained in Theorem 10.1 , but Theorem 6.1 states nothing in general concerning the second case.

The application of Theorems 9.1 and 9.2 to equation (10.1) yields

THEOREM 10.4. Let (10.2) and (10.3) hold. Assume that $p$ is neither 0 nor a real number greater than 1 . For some $\sigma, 1 \leqq \sigma \leqq 2$, let $\alpha(n)$ in (10.4) satisfy

$$
\sum^{\infty}|\alpha(n)|^{\sigma}<\infty \text {. }
$$

Then (10.1) has solutions $x_{ \pm}(n)$ satisfying respectively, as $n \rightarrow \infty$,

$$
x_{ \pm}(n)=\left(-\frac{1}{2}\right)^{n} \prod^{n-1}\left(a_{1}(k)(1 \pm v \mp \alpha(k) / 2 v)\right) .
$$

If $p$ is real and $p>1$, the conclusion remains valid if (10.7) is replaced by the stronger condition

$$
\sum^{\infty}|\alpha(n)|^{\sigma} n^{\tau-1}<\infty
$$

where $1 \leqq \sigma \leqq 2$ and $\tau>\sigma$.

The first case of this theorem (i.e., where $p$ real implies $p \neq 0$ and $p<1$ ) was proved by W. Ford [3] for $\alpha(n)$ having the form $\alpha(n)=\mu / n^{1 / 2+\delta}+\theta(n)$ where $0<\delta \leqq \frac{1}{2}$ and $\Sigma|\theta(n)|<\infty$. He also proves that if $\alpha(n)$ is of this form, $p$ is real, and $p>1$, so that $|1-v|=|1+v|$, then the result remains valid if 
$\left|1-v+\mu / 2 v n^{1 / 2+\delta}\right|=\left|1+v-\mu / 2 v n^{1 / 2+\delta}\right|$ for all large $n$, i.e., if $\mu$ is real.

Proof of Theorem 10.4. After making the change of variables (10.5), write (10.6) as a system

$$
\left(\begin{array}{l}
v^{1}(n+1) \\
v^{2}(n+1)
\end{array}\right)=\left(\begin{array}{ll}
0 & 1 \\
v^{2}-1-\alpha(n) & 2
\end{array}\right)\left(\begin{array}{l}
v^{1}(n) \\
v^{2}(n)
\end{array}\right),
$$

where $\left(v^{1}(n), v^{2}(n)\right)=(u(n), u(n+1))$. Put

$$
\left(\begin{array}{c}
w^{1}(n) \\
w^{2}(n)
\end{array}\right)=\frac{1}{2 v}\left(\begin{array}{cc}
1 & 1 \\
-(v-1) & v+1
\end{array}\right)\left(\begin{array}{l}
v^{1}(n) \\
v^{2}(n)
\end{array}\right)
$$

so that (10.9) transforms to

$$
\left(\begin{array}{l}
w^{1}(n+1) \\
w^{2}(n+1)
\end{array}\right)=\left(\begin{array}{ll}
1-v+\alpha(n) / 2 v & \alpha(n) / 2 v \\
-\alpha(n) / 2 v & 1+v-\alpha(n) / 2 v
\end{array}\right)\left(\begin{array}{l}
w^{1}(n) \\
w^{2}(n)
\end{array}\right) .
$$

The first part of the theorem follows by an application of Theorem 9.1 to (10.10).

If $p>1$, and (10.8) holds, then the system (10.10) satisfies the hypothesis of Theorem 9.2 ( $h_{0}$ may be taken to be $\tau / \sigma>1$, so (9.7) holds). The result follows by an application of that theorem.

Finally, as an analogue of Theorem 10.3, one has

THEOREM 10.5. Let (10.2) and (10.3) hold. Assume that $p=1$ and that $\alpha(n)$ in (10.4) satisfies $\Sigma^{\infty}|\alpha(n)|^{\sigma} n^{2 \sigma-1}<\infty$ for some $\sigma$ and $\tau$, where $1 \leqq \sigma \leqq 2$. Then (10.1) has solutions $x_{1}(n)$ and $x_{2}(n)$ satisfying respectively, as $n \rightarrow \infty$,

$$
\begin{aligned}
& x_{1}(n) \sim\left(-\frac{1}{2}\right)^{n}\left(\prod^{n-1} a_{1}(k)(1+(k+1) \alpha(k))\right), \\
& x_{2}(n) \sim\left(-\frac{1}{2}\right)^{n} n\left(\prod^{n-1} a_{1}(k)(1-k \alpha(k))\right) .
\end{aligned}
$$

Proof. Make the change of variables (10.5), then the resulting equation (10.6) is equivalent to the system

$$
\left(\begin{array}{l}
v^{1}(n+1) \\
v^{2}(n+1)
\end{array}\right)=\left(\begin{array}{cc}
1 & -\alpha(n) \\
1 & 1
\end{array}\right)\left(\begin{array}{l}
v^{1}(n) \\
v^{2}(n)
\end{array}\right)
$$

where $\left(v^{1}(n), v^{2}(n)\right)=(u(n+1)-u(n), u(n))$. Taking $h_{0}=\tau / \sigma$ as before, the hypothesis of Theorem 9.2 is satisfied by the above system. An application of that theorem implies that $(10.6)$ has solutions $u_{1}(n)$ and $u_{2}(n)$ satisfying respectively, as $n \rightarrow \infty$,

$$
u_{1}(n) \sim \prod^{n-1}(1+(k+1) \alpha(k)) \text { and } u_{2}(n) \sim n \prod^{n-1}(1-k \alpha(k)) .
$$

The conclusion of the theorem follows from (10.5). 


\section{REFERENCES}

1. O. Dunkel, Regular singular points of a system of homogeneous linear differential equations, Proc. Amer. Acad. Arts Sci. 38 (1902-03), 341-370.

2. M. Evgrabov, The asymptotic behavior of solutions of difference equations, Dokl. Akad. Nauk SSSR 121 (1958), 26-29. (Russian)

3. W. Ford, On the integration of the homogeneous linear difference equation of second order, Trans. Amer. Math. Soc. 10 (1909), 319-336.

4. A. Gelfond and I. Kubenskaya, On a theorem of Perron in the theory of difference equations, Izv. Akad. Nauk SSSR Ser. Mat. 17 (1953), 83-86. (Russian)

5. P. Hartman and A. Wintner, Asymptotic integrations of linear differential equations, Amer. J. Math. 77 (1955), 45-86.

6 - Asymptotic integrations of nonlinear differential equations, Amer. J. Math. 77 (1955), 692-724.

7. M. Nagumo, $A$ theory of degree of mapping based on infinitesimal analysis, Amer. J. Math. 73 (1951), 485-496.

8. C. Olech, On the asymptotic behavior of the solutions of a system of ordinary non-linear differential equations, Bull. Acad. Polon. Sci. Cl. III 4 (1956), 555-561.

9. O. Perron, Uber Stabilität und asymptotisches Verhalten der Integrale von Differentialgleichungssystem, Math. Z. 29 (1929), 129-160.

10. - Uber Stabilität und asymptotisches Verhalten der Losungen eines Systems endlicher Differenzengleichungen, J. Reine Angew. Math. 161 (1929), 41-64.

11. T. Ważewski, Sur un principe topologique de l'examen de l'allure asymptotique des intégrales des équations différentielles ordinaires, Ann. Soc. Polon. Math. 20 (1947), 279-313.

JOHNS HOPKINS UNIVERSITY,

BALTIMORE, MARYLAND 